\title{
Study of serum lipids as atherogenic risk factor in preclinical diabetes
}

\author{
Mukesh R Dinkar', Alpna Mathur 2,", Bharat H Patel ${ }^{3}$ \\ ${ }^{1}$ Professor and Head, Dept. of Physiology, GMERS Medical College, Vadodara, Gujarat, ${ }^{2}$ Associate Professor, Dept. of \\ Physiology, Government Medical College, Surat, Gujarat, ${ }^{3}$ Associate Professor, Dept. of Community Medicine, GMERS \\ Medical College, Vadodara, Gujarat, India
}

*Corresponding Author:

Email: alpnarishi@gmail.com

Received: $16^{\text {th }}$ February, 2018

Accepted: $26^{\text {th }}$ April, 2018

\begin{abstract}
Introduction and Objective: Preclinical diabetes has been associated with cardiovascular morbidity and mortality in Indian population. The current study was conducted to screen out preclinical diabetes (impaired fasting glycemia and impaired glucose tolerance) and assess serum lipids as atherogenic risk factor for future cardiovascular complications. We compared serum lipids in subjects with IFG and IGT to find out the high risk population so that early interventions can be done to prevent future cardiovascular morbidity and mortality.

Materials and Methods: 200 non-diabetic apparently healthy subjects were selected for the study. $75 \mathrm{~g}$ oral Glucose tolerance test (GTT) was performed in all of them, plasma glucose levels were estimated by GOD-POD method. Based on criteria by American Diabetic Association they were divided in four groups: Group I Normal fasting Glucose/Normal glucose tolerance (NFG/NGT, n=70), Group II isolated Impaired fasting glucose (iIFG, n=34), Group III isolated Impired glucose tolerance (iIGT, $\mathrm{n}=38$ ), Group IV Impaired fasting glucose and IGT(IFG/IGT, $n=54)$. Serum cholesterol, Triglycerides and HDL were estimated in the morning fasting samples from all the subjects and compared among the groups.

Results: The current study showed that Serum LDL and Triglycerides were significantly higher and serum HDL is significantly lower in prediabetic subjects (iIGT, iIGT, IFG/IGT) when compared with Control subjects (NFG/NGT). Also Serum LDL was significantly higher in those with iIGT and IFG/IGT as compared to those with iIFG thus increasing atherogenic risk in iIGT subjects. Also it was observed that Serum LDL, TG and HDL do not show significant changes between those having iIGT and IFG/IGT. Also these levels were comparable in those with iIFG and NGT. A positive correlation is also found between serum lipids and other known cardiovascular risk factors like BMI, Systolic blood pressure and Rate pressure product in prediabetic subjects.

Conclusion: This suggested that iIGT subjects having raised LDL, TG and low HDL were at a higher risk of atherogenesis and future coronary heart diseases (CHD), hence can be targeted for early dietery, lifestyle and pharmacological interventions to prevent future risk of CHD.
\end{abstract}

Keywords: Impaired fasting glycemia (IFG), Impaired glucose tolerance (IGT), Low density lipoprotein (LDL), Triglycerides (TG), High density Lipoprotein (HDL), Coronary Heart Disease (CHD).

\section{Introduction}

India is home to 72 million diabetics, second only to China which is home to 114 million diabetics in 2017 as per data of International Diabetes Federation. IFG and IGT are preclinical conditions to diabetes mellitus which along with its fatal macrovascular complications mainly coronary heart disease (CHD) is one of the leading causes of mortality and morbidity in the Indian population. Hence it is important to screen the population for preclinical diabetes by performing oral Glucose Tolerance Test (GTT) and assessing the risk factors for future diabetes and its cardiovascular complications at an early stage so that necessary lifestyle, dietary and medical interventions can be done to prevent future morbidity and mortality. Though both IFG and IGT are risk factors for future DM and coronary heart disease, IGT has been more consistently associated with metabolic syndrome increasing risk for CHD in various studies. ${ }^{1-4}$ However association of IFG with future cardiovascular complications is still unclear. ${ }^{1,5-7}$ There are evidences showing that decreased HDL and raised LDL and triglycerides have been consistently associated with increased risk of CHD by increasing plaque formation, inflammation, endothelial damage, thrombosis and affecting vasomotion. ${ }^{8,9}$ In the current study we have attempted to find out serum lipids as atherogenic risk factor for future CHD in various prediabetic groups (iIFG, iIGT, IFG/IGT) to identify the high risk population. Further we have tried to find out the correlation between serum lipids and other cardiovascular risk factors like body mass index (BMI), systolic blood pressure, plasma glucose and Rate pressure product (RPP) in various prediabetic groups.

\section{Materials and Methods}

Study Design: A cross sectional study was conducted on 200 non diabetic apparently healthy subjects aged 30-70 years. A detailed history and examination was done and subjects with history of known cardiovascular disorders, liver and renal dysfunction, thyroid disease, pregnant and nursing females, smokers and those with any acute or chronic illness were excluded from the study. An informed written consent was obtained from 
all the participants and details of procedure were explained to them in the local language. Institutional Ethics committee approval was obtained and as per the guidelines by World Medical Declaration of Helsinki study was conducted.

Glucose and lipid estimation and classification of subjects: After an overnight fasting morning blood samples were collected for fasting plasma glucose and lipid estimations followed by $75 \mathrm{~g}$ oral GTT. Four subjects with fasting plasma glucose (FPG)>126mg\% and 2 hour post challenge glucose levels $(2 \mathrm{hPG})>200 \mathrm{mg} \%$ were defined to have diabetes and excluded from the study. Serum cholesterol was estimated by cholesterol oxidase peroxidase method, triglycerides were estimated by GPO-ESPAS enzymatic method and HDL by HDL-PEG-PAP precipitation method using Glaxo estimation kits. LDL was calculated using Friedewald's formula. All biochemical estimations were done by Miura biochemical fully automatic (Trans Asia biomedical limited model: Erba XL-640, Mumbai) in the biochemistry laboratory, GMC Surat.

Based on criteria by American Diabetic Association, the subjects were divided into four groups based on their FPG and 2hPG values as follows:

Group I: normal fasting glucose/normal glucose tolerance (NFG/NGT) with FPG $<100 \mathrm{mg} \%$ and $2 \mathrm{hPG}<140 \mathrm{mg} \%$.

Group II: Isolated IFG (iIFG) with FPG $>100 \mathrm{mg} \%$ but $<126 \mathrm{mg} \%$ and $2 \mathrm{hPG}<140 \mathrm{mg} \%$.
Group III: Isolated impaired glucose tolerance (iIGT) with FPG $<100 \mathrm{mg} \%$ and $2 \mathrm{hPG}>140 \mathrm{mg} \%$ but less than $200 \mathrm{mg} \%$.

Group IV: IFG/IGT with FPG $>100 \mathrm{mg} \%$ but $<126 \mathrm{mg} \%$ and $2 \mathrm{hPG}>140 \mathrm{mg} \%$ but $<200 \mathrm{mg} \%$.

Other Parameters: Height and weight were measured to calculate BMI using formula weight $(\mathrm{kg}) / \mathrm{height}\left(\mathrm{m}^{2}\right)$. Heart rate $(\mathrm{HR})$ and $\mathrm{BP}$ were measured after 10 minutes of supine rest. Rate pressure product (RPP), which is an index of myocardial risk factor and a known cardiovascular risk parameter was calculated using formula:

RPP = HR x systolic BP/100.

\section{Statistical Analysis of Data}

Statistical software SPSS version 16 was used for statistical analysis of data. Serum HDL, TG, LDL, BMI, FPG, 2hPG, SBP, DBP and RPP were expressed as Mean \pm Standard deviation. For the purpose of statistical analysis, data were assessed for normal distribution and variables were compared in the four group using one way ANOVA and post-hoc test. Pearson's correlation analysis was done to find out correlation between serum TG, HDL and LDL with other variables like BMI, SBP and RPP. Simple linear regression analysis was done using Serum TG and LDL as dependent variable and RPP, a known cardiovascular risk factor as independent variable in all the four groups. $\mathrm{P}$ value $<0.05$ was considered as statistically significant.

\section{Results}

Table 1 Age, BMI and cardiovascular parameters (Systolic BP, Diastolic BP, Heart rate and RPP) among the groups

\begin{tabular}{|l|c|c|c|c|c|}
\hline \multicolumn{1}{|c|}{ Variables } & NFG/NGT & iIFG & iIGT & IFG/IGT & p \\
\hline $\mathrm{n}$ & 70 & 34 & 38 & 54 & - \\
\hline Age $(\mathrm{years})$ & $48.6 \pm 8.6$ & $50.6 \pm 9.8$ & $51.1 \pm 9.1$ & $54.8 \pm 8.6^{* * *, \#}$ & 0.002 \\
\hline BMI $\left(\mathrm{kg} / \mathrm{m}^{2}\right)$ & $21.3 \pm 3.1$ & $22.7 \pm 3.1$ & $24 \pm 3.9^{* *}$ & $24.5 \pm 4.7^{* * *}, \#$ & 0.000 \\
\hline SBP $(\mathrm{mmHg})$ & $121.3 \pm 9.0$ & $121.5 \pm 11.1$ & $124.9 \pm 9.9$ & $126.4 \pm 11.1^{* *}, \#$ & 0.023 \\
\hline DBP $(\mathrm{mmHg})$ & $79.2 \pm 5.9$ & $80.0 \pm 6.3$ & $80.7 \pm 6.1$ & $82.2 \pm 7.0^{*}$ & 0.075 \\
\hline Heart rate $(\mathrm{bpm})$ & $74.2 \pm 6.8$ & $85.1 \pm 7.1^{* * *}$ & $86.1 \pm 8.1^{* * *}$ & $89.0 \pm 10.6^{* * * * \#}$ & 0.000 \\
\hline RPP $(\mathrm{mmHg} / \mathrm{min})$ & $90.2 \pm 12.3$ & $103.5 \pm 13.6^{* * *}$ & $107.4 \pm 12.3^{* * *}$ & $113.1 \pm 20.8^{* * *, \# \#}$ & 0.000 \\
\hline
\end{tabular}

(The $p<0.05$ was statistically considered significant. The * mark indicates comparison with NFG/NGT group and the \# mark indicates comparison with iIFG group. ${ }^{*} p<0.05, * * p<0.01, * * * p<0.001,{ }^{\#} p<0.05,{ }^{\# \#} p<0.01,{ }^{\# \#} p<0.001$. NFG/NGT: Normal fasting glucose/normal glucose tolerance, iIFG: Isolated impaired fasting glucose, iIGT: Isolated impaired glucose tolerance, IFG/IGT: Impaired fasting glycemia/impaired glucose tolerance, BMI: Body mass index, SBP: Systolic blood pressure, DBP: Diastolic blood pressure, RPP: Rate pressure product)

Table 2: Fasting plasma glucose, 2 hour plasma glucose and Serum lipids (HDL, LDL and Triglycerides) among the groups

\begin{tabular}{|l|c|c|c|c|}
\hline \multicolumn{1}{|c|}{ Variables } & $\begin{array}{c}\text { NFG/NGT } \\
(\mathbf{n = 7 0})\end{array}$ & $\begin{array}{c}\text { iIFG } \\
(\mathbf{n = 3 4 )}\end{array}$ & $\begin{array}{c}\text { iIGT } \\
(\mathbf{n = 3 8})\end{array}$ & $\begin{array}{c}\text { IFG/IGT } \\
(\mathbf{n = 5 4})\end{array}$ \\
\hline FPG $(\mathrm{mg} \%)$ & $84.3 \pm 8.4$ & $113.9 \pm 7.2^{* * *}$ & $89.4 \pm 6.0^{* *}, \# \#, \dagger \dagger \dagger$ & $114.8 \pm 6.3^{* * * *}$ \\
\hline 2 h PG $(\mathrm{mg} \%)$ & $119.0 \pm 11.6$ & $129.1 \pm 6.5^{* * *}$ & $164.4 \pm 16.0^{* * *}, \# \# \#$ & $168.6 \pm 15.9^{* * *}$ \\
\hline HDL $(\mathrm{mg} / \mathrm{dl})$ & $45.2 \pm 9.2$ & $41.3 \pm 7.4^{*}$ & $41.5 \pm 8.5^{*}$ & $42.2 \pm 7.4^{*}$ \\
\hline
\end{tabular}




\begin{tabular}{|l|c|c|c|c|}
\hline $\mathrm{LDL}(\mathrm{mg} / \mathrm{dl})$ & $117.7 \pm 32.2$ & $119.2 \pm 30.8$ & $135.1 \pm 33.9^{* *, \#}$ & $133.9 \pm 23.9^{* *, \#}$ \\
\hline TG $(\mathrm{mg} / \mathrm{dl})$ & $129.5 \pm 34.9$ & $132.8 \pm 31.0$ & $149.9 \pm 35.9^{* *}$ & $151.5 \pm 28.3^{* * *}$ \\
\hline
\end{tabular}

(The $\mathrm{p}<0.05$ was statistically considered significant. The * mark indicates comparison with NFG/NGT group, the \# mark indicates comparison with iIFG group and $\dagger$ mark indicates comparison with ilGT group. ${ }^{*} p<0.05, * * p<0.01$, ${ }^{* * *} p<0.001,{ }^{\#} p<0.05,{ }^{\#} p<0.01,{ }^{\# \#} p<0.001,{ }^{\dagger} \mathrm{p}<0.05,{ }^{\dagger \dagger} \mathrm{p}<0.01,{ }^{\dagger \dagger} \mathrm{p}<0.001$. NFG/NGT: Normal fasting glucose/normal glucose tolerance, iIFG: Isolated impaired fasting glucose, iIGT: Isolated impaired glucose tolerance, IFG/IGT: Impaired fasting glycemia/impaired glucose tolerance, WBC: White blood cell, FPG: Fasting plasma glucose, 2 h P: 2 hour post-challenge glucose values.)

Table 3: Bivariatecorrelation of serum HDL with BMI, SBP, RPP in four groups

\begin{tabular}{|l|c|c|c|c|c|c|c|c|}
\hline & \multicolumn{2}{|c|}{ NFG/NGT $(\mathbf{n = 7 0})$} & \multicolumn{2}{c|}{ iIFG $(\mathbf{n = 3 4 )}$} & \multicolumn{2}{c|}{ iIGT $(\mathbf{n = 3 8})$} & \multicolumn{2}{c|}{ IFG/IGT $(\mathbf{n = 5 4})$} \\
\cline { 2 - 9 } & $\mathbf{r}$ & $\mathbf{p}$ & $\mathbf{r}$ & $\mathbf{p}$ & $\mathbf{R}$ & $\mathbf{p}$ & $\mathbf{r}$ & $\mathbf{P}$ \\
\hline BMI & -0.18 & 0.13 & 0.08 & 0.66 & -0.05 & 0.76 & 0.13 & 0.34 \\
\hline SBP & -0.19 & 0.11 & 0.14 & 0.42 & 0.13 & 0.44 & 0.24 & 0.08 \\
\hline RPP & -0.22 & 0.07 & 0.16 & 0.36 & -0.29 & 0.08 & 0.18 & 0.19 \\
\hline
\end{tabular}

$\left({ }^{*} \mathrm{p}<0.05\right.$ was statistically considered significant. NFG/NGT: Normal fasting glucose/normal glucose tolerance, iIFG: Isolated impaired fasting glucose, iIGT: Isolated impaired glucose tolerance, IFG/IGT: Impaired fasting glucose/impaired glucose tolerance, BMI: Body mass index, SBP: Systolic blood pressure, RPP: Rate pressure product)

Table 4: Bivariatecorrelation of serum LDL with BMI, SBP, RPP in four groups

\begin{tabular}{|l|c|c|c|c|c|c|c|c|}
\hline \multirow{2}{*}{} & \multicolumn{2}{|c|}{ NFG/NGT $(\mathbf{n = 7 0})$} & \multicolumn{2}{c|}{ iIFG $(\mathbf{n}=\mathbf{3 4})$} & \multicolumn{2}{c|}{ iIGT (n=38) } & \multicolumn{2}{c|}{ IFG/IGT (n=54) } \\
\cline { 2 - 8 } & $\mathbf{r}$ & $\mathbf{p}$ & $\mathbf{r}$ & $\mathbf{p}$ & $\mathbf{R}$ & $\mathbf{p}$ & $\mathbf{r}$ & $\mathbf{P}$ \\
\hline BMI & 0.44 & $0.00^{*}$ & 0.78 & $0.00^{*}$ & 0.34 & $0.03^{*}$ & 0.32 & 0.12 \\
\hline SBP & 0.46 & $0.00^{*}$ & 0.36 & $0.04^{*}$ & 0.53 & $0.00^{*}$ & 0.36 & $0.01^{*}$ \\
\hline RPP & 0.46 & $0.00^{*}$ & 0.33 & 0.06 & 0.39 & $0.02^{*}$ & 0.34 & $0.01^{*}$ \\
\hline
\end{tabular}

$\left({ }^{*} \mathrm{p}<0.05\right.$ was statistically considered significant. NFG/NGT: Normal fasting glucose/normal glucose tolerance, iIFG: Isolated impaired fasting glucose, iIGT: Isolated impaired glucose tolerance, IFG/IGT: Impaired fasting glucose/impaired glucose tolerance, BMI: Body mass index, SBP: Systolic blood pressure, RPP: Rate pressure product)

Table 5: Bivariatecorrelation of serum TG with BMI, SBP, RPP in four groups

\begin{tabular}{|l|c|c|c|c|c|c|c|c|}
\hline & \multicolumn{2}{|c|}{ NFG/NGT $(\mathbf{n}=\mathbf{7 0})$} & \multicolumn{2}{c|}{ iIFG $(\mathbf{n}=\mathbf{3 4})$} & \multicolumn{2}{c|}{ iIGT $(\mathbf{n}=\mathbf{3 8})$} & \multicolumn{2}{c|}{ IFG/IGT $(\mathbf{n = 5 4})$} \\
\cline { 2 - 9 } & $\mathbf{r}$ & $\mathbf{p}$ & $\mathbf{r}$ & $\mathbf{p}$ & $\mathbf{R}$ & $\mathbf{p}$ & $\mathbf{r}$ & $\mathbf{P}$ \\
\hline BMI & 0.48 & $0.00^{*}$ & 0.67 & $0.00^{*}$ & 0.26 & 0.12 & 0.32 & $0.02^{*}$ \\
\hline SBP & 0.41 & $0.00^{*}$ & 0.37 & $0.03^{*}$ & 0.36 & $0.03^{*}$ & 0.38 & $0.01^{*}$ \\
\hline RPP & 0.44 & $0.00^{*}$ & 0.42 & $0.01^{*}$ & 0.49 & $0.00^{*}$ & 0.33 & $0.02^{*}$ \\
\hline
\end{tabular}

$\left({ }^{*} \mathrm{p}<0.05\right.$ was statistically considered significant. NFG/NGT: Normal fasting glucose/normal glucose tolerance, iIFG: Isolated impaired fasting glucose, iIGT: Isolated impaired glucose tolerance, IFG/IGT: Impaired fasting glucose/impaired glucose tolerance, BMI: Body mass index, SBP: Systolic blood pressure, RPP: Rate pressure product)

Table 6: Simple linear regression analysis with serum LDL as dependent variable and RPP as independent variable in all 4 groups

\begin{tabular}{|c|c|c|c|c|c|c|}
\hline \multirow[t]{2}{*}{ Group } & \multirow[t]{2}{*}{ Model } & \multicolumn{2}{|c|}{$\begin{array}{l}\text { Unstandardized } \\
\text { Coefficients }\end{array}$} & \multirow{2}{*}{$\begin{array}{c}\begin{array}{c}\text { Standardized } \\
\text { Coefficients }\end{array} \\
\text { Beta }\end{array}$} & \multirow[t]{2}{*}{$\mathbf{t}$} & \multirow[t]{2}{*}{ Significance } \\
\hline & & B & Std. Error & & & \\
\hline \multirow{3}{*}{$\begin{array}{l}\text { NFG/NGT } \\
(\mathrm{n}=70)\end{array}$} & Constant & 8.27 & 25.66 & & 0.32 & 0.75 \\
\hline & RPP & 1.21 & 0.28 & 0.46 & 4.30 & $0.00 *$ \\
\hline & \multicolumn{6}{|c|}{ Regression Equation: Serum LDL= $8.27+1.21$ (RPP) } \\
\hline \multirow{3}{*}{$\begin{array}{l}\text { iIFG } \\
(n=34)\end{array}$} & Constant & 41.87 & 39.42 & & 1.06 & 0.30 \\
\hline & RPP & 0.75 & 0.38 & 0.33 & 1.98 & 0.06 \\
\hline & \multicolumn{6}{|c|}{ Regression Equation: Serum LDL $=41.87+0.75(\mathrm{RPP})$} \\
\hline \multirow{3}{*}{$\begin{array}{l}\text { iIGT } \\
(n=38)\end{array}$} & Constant & 18.68 & 45.83 & & 0.41 & 0.69 \\
\hline & RPP & 1.08 & 0.42 & 0.39 & 2.56 & $0.02 *$ \\
\hline & \multicolumn{6}{|c|}{ Regression Equation: Serum LDL= $18.68+1.08$ (RPP) } \\
\hline
\end{tabular}




\begin{tabular}{|l|c|c|c|c|c|c|}
\hline \multirow{2}{*}{$\begin{array}{l}\text { IFG/IGT } \\
(\mathrm{n}=54)\end{array}$} & Constant & 89.20 & 17.22 & & 5.18 & $0.00^{*}$ \\
\cline { 2 - 7 } & RPP & 0.40 & 0.15 & 0.34 & 2.64 & $0.01^{*}$ \\
\cline { 2 - 6 } & \multicolumn{2}{|l|}{ Regression Equation: Serum LDL $=89.20+0.40$ (RPP) } \\
\hline
\end{tabular}

$\left({ }^{*} \mathrm{p}<0.05\right.$ was statistically considered significant. RPP: Rate pressure product, NFG/NGT: Normal fasting glucose/normal glucose tolerance, iIFG: Isolated impaired fasting glucose, iIGT: Isolated impaired glucose tolerance, IFG/IGT: Impaired fasting glucose/impaired glucose tolerance)

Table 7: Simple linear regression analysis with serum TG as dependent variable and RPP as independent variable in all 4 groups

\begin{tabular}{|c|c|c|c|c|c|c|}
\hline \multirow[t]{2}{*}{ Group } & \multirow[t]{2}{*}{ Model } & \multicolumn{2}{|c|}{$\begin{array}{c}\text { Unstandardized } \\
\text { Coefficients }\end{array}$} & \multirow{2}{*}{$\begin{array}{c}\begin{array}{c}\text { Standardized } \\
\text { Coefficients }\end{array} \\
\text { Beta } \\
\end{array}$} & \multirow[t]{2}{*}{$\mathbf{t}$} & \multirow[t]{2}{*}{ Significance } \\
\hline & & B & Std. Error & & & \\
\hline \multirow{3}{*}{$\begin{array}{l}\text { NFG/NGT } \\
(n=70)\end{array}$} & Constant & 15.85 & 28.19 & & 0.56 & 0.58 \\
\hline & RPP & 1.26 & 0.31 & 0.44 & 4.07 & $0.00 *$ \\
\hline & \multicolumn{6}{|c|}{ Regression Equation: Serum LDL= $15.85+1.26(\mathrm{RPP})$} \\
\hline \multirow{3}{*}{$\begin{array}{l}\text { iIFG } \\
(\mathrm{n}=34)\end{array}$} & Constant & 34.49 & 38.28 & & 0.90 & 0.37 \\
\hline & RPP & 0.95 & 0.37 & 0.42 & 2.59 & $0.01 *$ \\
\hline & \multicolumn{6}{|c|}{ Regression Equation: Serum LDL= $34.49+0.95$ (RPP) } \\
\hline \multirow{3}{*}{$\begin{array}{l}\text { iIGT } \\
(\mathrm{n}=38)\end{array}$} & Constant & -4.71 & 45.86 & & -0.10 & 0.92 \\
\hline & RPP & 1.44 & 0.42 & 0.49 & 3.39 & $0.00 *$ \\
\hline & \multicolumn{6}{|c|}{ Regression Equation: Serum LDL= -4.71 + 1.44 (RPP) } \\
\hline \multirow{2}{*}{$\begin{array}{l}\text { IFG/IGT } \\
(\mathrm{n}=54)\end{array}$} & Constant & 101.11 & 20.52 & & 4.93 & $0.00^{*}$ \\
\hline & RPP & 0.45 & 0.18 & 0.33 & & $0.02 *$ \\
\hline
\end{tabular}

( ${ }^{\mathrm{p}}<0.05$ was statistically considered significant. RPP: Rate pressure product, NFG/NGT: Normal fasting glucose/normal glucose tolerance, iIFG: Isolated impaired fasting glucose, iIGT: Isolated impaired glucose tolerance, IFG/IGT: Impaired fasting glucose/impaired glucose tolerance)

Table 1 depicts mean and standard deviation of different variables in all four groups. Age of the subjects was comparable in all the four groups (except in the IFG/IGT). BMI was significantly higher in iIGT and IFG/IGT as compared to NFG/NGT and iIFG groups. There was no significant difference among four groups for SBP and DBP (except in the IFG/IGT). HR and RPP were significantly higher in pre-diabetic subjects compared to NFG/NGT group.

Table 2 depicts that serum HDL level was significantly lower in pre-diabetics as compared to NFG/NGT group. While, serum LDL and TG level was significantly higher in iIGT and IFG/IGT group compared to NFG/NGT group. Further, the serum LDL level was significantly higher in iIGT and IFG/IGT groups compared to iIFG group.

Pearson's correlation analysis depicts that there was no significant correlation of BMI, SBP and RPP with serum HDL in all four groups. While BMI, SBP and RPP had significant positive correlation with serum LDL and serum TG in all four groups, more so in NFG/NGT group (Exception: no significant correlation of serum LDL with BMI in IFG/IGT group and serum LDL with RPP in iIFG group) [Table 3, 4, 5].

Table 6 depicts simple linear regression of serum LDL and RPP and table 7 depicts simple linear regression of serum TG with RPP. Both LDL and TG were found to be an independent atherogenic risk factor for future cardiovascular complications in all four groups except LDL in iIFG group.

\section{Discussion}

Dyslipidemias characterized by raised triglycerides (TG) and LDL and decreased HDL have been positively associated with plaque formation, endothelial dysfunction, atherogenesis and future cardiovascular complications..$^{9-13}$ In prediabetics due to insulin resistance oxidized LDL and triglycerides rich lipoproteins increases which leads to endothelial accumulation and uptake by macrophages to form foam cells which promotes fatty streak formation. ${ }^{11,14}$ Also they enhance recruitment and attachment of monocytes by increasing Tumour Necrotic Factor-alpha(TNF), Interleukin-1beta(IL-1), chemotactic proteins, cellular adhesion molecule-1and matrix metalloproteinase-3 which decreases flow mediated vasodilatationthus leading to atherosclerosis. ${ }^{9}$ They also suppress atheroprotective and anti-inflammatory effects of HDL by blocking sterole efflux from monocytes. Various studies have shown that dyslipedemias and other cardiovascular risk factors like hsCRP, IL-1, WBCs and other inflammatory markers start increasing in prediabetics even before onset of clinically evident diabetes. ${ }^{11}$ Studies have shown increased cardiovascular risk factors like elevated Resting heart rate, rate pressure product (RPP), decreased heart rate variability (HRV) in first degree relatives of type $2 \mathrm{DM}$. Hence the 
current study has been undertaken to study the lipid profile in prediabetics and compare Serum LDL, TG and HDL in IFG and IGT groups to assess the atherogenic risk profile and find out the high risk group for future cardiovascular complications, so that early interventions can be done to prevent future risk of Coronary heart disease in them. Also we have attempted to study the association between serum lipids and other cardiovascular risk factors like raised BMI, Blood pressure and Rate pressure product in various prediabetic groups. It was found that BMI, Heart rate, pulse pressure and serum LDL and Triglycerides were significantly higher in prediabetics as compared to control. Also serum LDL was found to be significantly higher in iIGT and IFG/IGT groups as compared to iIFG group. Correlation analysis showed that serum LDL and TG were significantly positively associated with BMI, SBP, RPP in all four groups except that no correlation of LDL and BMI is found in IFG/IGT groups and LDL with RPP in iIFG group. Linear regression analysis showed that serum TG and LDL are independent risk factors for future cardiovascular complications with RPP (a known cardiovascular risk factor) as independent variable in all four groups except for LDL in iIFG group.

Various previous studies have distinctly associated prediabetes (defined as IFG and IGT) with future risk of coronary heart disease (CHD). Tominga et al (1999) in Fungata diabetes study, ${ }^{2}$ DECODE study group $(2001)^{3}$ and Blake et al $(2004)^{1}$ have positively associated IGT with increased risk of CHD in western population. Pan et al(1993) found similar associations in Chinese population. ${ }^{4}$ However studies associating IFG with future $\mathrm{CHD}$ showed conflicting results Levitzky et al(2008) and Kim et al(2013) have positively associated IFG with risk of CHD in women and men respectively. ${ }^{5,6}$ Whereas Blake et al(2004)and Hashemi et al(2008)have not found any correlation between IFG and increased risk of CHD. ${ }^{1,7}$ In 1990 Haffner et al found raised LDL, TG, fasting glucose, insulin, BMI, BP and decreased HDL in prediabetics in Mexican population and attributed this atherogenic risk profile to insulin resistance and hyperinsulinemia. ${ }^{14}$ Chakarova et al (2009) associated both IFG and IGT with increased cardiovascular risk which is characterised by significant atherogenic changes in serum lipids and hsCRP. ${ }^{11}$ Also they found that those with IGT has significantly higher TG, free fatty acids and lower HDL-c as compared to IFG group making it a higher prediabetic risk group for future cardiovascular complications similar to newly diagnosed Diabetics. These findings are consistent with our results. Wien et al (2010) suggested a 16 week ADA diet with $20 \%$ energy derived from almonds (20 oz per day) to improve markers of insulin sensitivity in prediabetics thus reducing the risk of atherogenesis in them. ${ }^{16}$ Thiyagarajan et al(2012) found elevated heart rate, altered cardiac autonomic functions, raised total cholesterol, LDL-c, TG to HDL-c ratio, oxidative stress and inflammatory markers like hs CRP, TNF alpha in IFG subjects as compared to control making them more prone for future cardiovascular complications in Indian population. ${ }^{17}$ Similar results were found in our study too. Lorenzo et al(2013) difference in lipoprotein and apolipoprotein B by a more sophisticated method like nuclear magnetic resonance(NMR) spectroscopy in IFG and IGT group and found increased cardiovascular risk in IGT group due to difference in degree of insulin resistance which also supports our findings. ${ }^{18}$ Kansal et al (2016) also screened population for prediabetes (IFG/IGT) by performing Glucose tolerance test(GTT) and found that total cholesterol(TC), LDL, TG, VLDL, TG/HDL ratio were significantly higher in prediabetics and suggested pharmacotherapy and lifestyle modifications in them to decrease future cardiovascular risk in Indian population. ${ }^{19}$

Limitations: Specific inflammatory markers like hsCRP, TNF alpha etc, oxidative stress markers and cardiac autonomic function tests have not been done in the current study to specifically define the higher cardiovascular risk group. Therefore further longitudinal follow up studies should be conducted on larger population to predict and control atherogenic risk in prediabetic population.

\section{Conclusion}

With the established fact that prediabetics are having higher risk of future cardiovascular morbidity as compared to normal population, it can be concluded from our study that iIGT and IFG/IGT groups have a significantly higher atherogenic risk profile due to difference in insulin sensitivity as compared to iIFG group amongst prediabetics. Hence it is important to screen out prediabetics from general population by performing oral GTT so that we can identify the high risk group (iIGT and IFG/IGT) and early dietery, lifestyle and pharmacological interventions can be done in them to prevent future cardiovascular morbidity and mortality in Indian population.

Acknowledgement: I am thankful to Department of clinical chemistry for their support and guidance. My heartiest gratitude towards all me subjects without whom this wouldn't have been possible.

\section{Conflict of Interest: Nil}

\section{References}

1. Blake DR, Meigs JB, Muller DC, Najjar SS et al. IGT but not IFG is associated with increased levels of CHD risk factors: results from Baltimore longitudinal study on ageing. Diabetes. 2004;53(8):2095-100.

2. Tominga M, Eguchi H, Manaka H, Igarash K, Kato T, Sekikwa A. IGT is a risk factor for CVD, but not IGT. The Fungata Diabetes study. Diabetes Care. 1999;22(6):920-4. 
3. DECODE study group. Glucose Tolerance and cardiovascular mortality: Comparison of fasting and $2 \mathrm{hr}$ diagnostic criteria. Arch intern Med. 2001;161(3):397405.

4. Pan XR, Hu YH, Li GW, Liu PA, Bennett PH, Howard BV.IGT and its relationship to ECG indicated CHD and risk factors among Chinese.Da Qing IGT and Diabetes study. Diabetes Care. 1993;16(1):150-6.

5. Levitzky JS, Pencina MJ,D’ Agostino RB et al. Impact of IFG on CVD. The Framingham Heart study. $J$ Am Coll Cardiol. 2008;51(3):264-270.

6. Kim HK, Kim CH, Kim EH, Base SJ et al. IFG and risk of CVD in Korean Men and women: The Korean Heart study. Diabetes care. 2013;36(2):328-35.

7. Hashemi M, Taheri H, Amiri N, Yavari M et al. Is IFG associated with increased risk of coronary arteriosclerosis. Acta Medica Iranica. 2008;46(1):38-42.

8. Mohan V, Deepa R, Subramaniam SR, Gopal P. Prevelence of coronary artery disease and its relationship with lipids in selected population in south India: The Chennai Urban Population Study no. 5. Journal of American College of Cardiology 2001;38(3):682-87.

9. Witztum JL, Steinberg D. Role of oxidized LDL in atherogenesis. Journal Clin. Invest.1991;88:1785-92.

10. Chen $\mathrm{AH}$, Tseng $\mathrm{CH}$. The role of Triglycerides in cardiovascular disease in Asian patients with Type 2 diabetes-A systematic Review. The Review of Diabetic Studies. 2013;10(2-3):101-109.

11. Chakarova N, Tankova T, Attanassova I, Dakovska L. Serum lipid and hsCRP levels in prediabetes--impaired fasting glucose (IFG) and impaired glucose tolerance (IGT). Diabetes Res Clin Pract. 2009;86(1):55-60.

12. 12) Manninen V, Tenkanen L, Koshinen P, Huttunen JK, Manttari M, Heinonen OP, Frick MH. Joint effects of serum TG and LDL cholesterol and HDL cholesterol on coronary heart disease risk in Helsinki Heart study: Implications for treatment. Circulation. 1992;85:37-45.

13. Jeppesen J, Heino HO, Suadicani P, Gyntelberg F.Relation of high TG-low HDL cholesterol and LDL cholesterol to the incidence of IHD An 8 year follow up in Copenhagen Male study. Arteriosclerosis Thrombosis Vascular Biology. 1997;17(6):1114-20.

14. Haffner SM, Stern MP, Hazuda HP, Mitchell BD, Patterson JK. Cardiovascular risk factors in confirmed
Prediabetic individuals. Does the clock for CHD starts ticking before onset of clinical Diabetes. JAMA. 1990;263(21):2893-98.

15. Pal GK, Adithan C, Dutta TK, Pal P et al. Association of hypertension status and cardiovascular risk with sympathovagal imbalance in first degree relatives of type 2 diabetes. Journal of diabetes investigation. 2014;5(4):449-55.

16. Wien M, Bleich D, Raghuwanshi M, Gould-Forgerite S, Gomes J, Monahan-Couch L, Oda K. Almond consumption and cardiovascular risk factors in adults with prediabetes. Journal of Am Coll of Nutrition. 2010;29(3):189-97.

17. Thiyagarajan R, Subramanian SK, Sampath N, trakroo M, Pal P, Bobby Z, Paneersevlam S, Das AK. Association between Cardiac Autonomic Function, Oxidative Stress and Inflammatory Response in Impaired Fasting Glucose Subjects: Cross-Sectional

Study.2012.http://journals.plos.org/plosone/article?id=10. 1371/journal.pone.0041889

18. Lorenzo C, Hartnett S, Hanley AJ, Rewers MJ, Impaired Fasting Glucose and Impaired Glucose Tolerance Have Distinct Lipoprotein and Apolipoprotein Changes: The Insulin Resistance Atherosclerosis Study. The Journal of clinical endocrinology and Metabolism. 2013;98(4):1622-30.

19. Kansal S, Kamble TK. Lipid Profile in Prediabetes. Journal of Association of Physicians of India. 2016;64:19-21.

How to cite this article: Dinkar MR, Mathur A, Patel BH. Study of serum lipids as atherogenic risk factor in preclinical diabetes. Ind $\mathbf{J}$ Clin Anat Physiol. 2018;5(3):320-325. 